\title{
Active Aerodynamic Control of a Separated Flow Using Streamwise Synthetic Jets
}

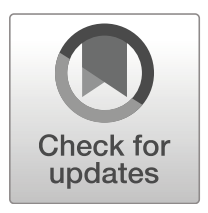

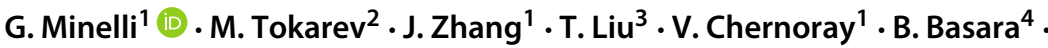 \\ S. Krajnović ${ }^{1}$
}

Received: 5 March 2019 / Accepted: 31 July 2019 / Published online: 30 August 2019

(C) The Author(s) 2019

\begin{abstract}
LES simulations at $R e=1 \times 10^{5}$ and wind tunnel experiments at $R e=5 \times 10^{5}$ were conducted to investigate the beneficial effect of an active flow control (AFC) technique on the aerodynamic performance of a simplified truck geometry. The paper involves the investigation of a synthetic jet actuator characterized by periodic blowing and suction that defines a zero net mass flux flow control mechanism. The actuation aims to suppress the flow separation occurring at the A-pillar (front rounded corner) of a truck cabin. The work flow is defined as it follows. First, LES at low Reynolds number are conducted for different disposition of the actuation slots. The results show a beneficial effect when the actuation slots are positioned in streamwise direction compared to spanwise (vertical) direction. Second, based on the previous considerations, wind tunnel experiments are conducted to verify and support the numerical findings. Both numerical solutions and experimental data show the same trend and the superiority of the streamwise slots actuation when compared to traditional vertical slot actuation. In particular, this work shows the weakness of a vertical slot actuation, when its location is not optimized. A small change in its positioning greatly worsen the efficacy of the separation control in terms of drag reduction and separation bubble length. The slot location directly affects the length of the separated flow region which its reduction can vary between $40-70 \%$ based on the positioning. Conversely, a streamwise actuation, spanning a larger portion of the curvature of a rounded A-pillar, is not affected by this behaviour and contributes up to $80 \%$ of the recirculation bubble reduction measured in the unactuated case. The effect of the location change and the orientation of a zero net mass flux jet slot is therefore investigated and discussed in this work.
\end{abstract}

Keywords Flow control - Synthetic jets · Drag reduction - Bluff body aerodynamics · Vehicle aerodynamics

G. Minelli

minelli@chalmers.se

1 Department of Mechanics and Maritime Sciences (M2), Chalmers University of Technology, 412 96, Gothenburg, Sweden

2 Institute of Thermophysics SB RAS, Novosibirsk, Russia

3 Key Laboratory of Traffic Safety on Track of Ministry of Education, School of Traffic, Transportation Engineering, Central South University, Changsha, 410075, China

4 AVL List GmbH, Advanced Simulation Technologies, Hans-List-Platz 1, 8020, Graz, Austria 


\section{Introduction}

The suppression of separated flow regions around road vehicles is key to reduce their aerodynamic drag and optimize their power consumption. In fact, more than $60 \%$ of the total power generated by a road vehicle is needed to overcome drag at cruise condition. The aerodynamic drag of a truck is in fact dominated by pressure drag due to heavily separated flow ( $90 \%$ of the total aerodynamic drag) and only a small percentage is attributed to the skin friction resistance [1]. Many are the regions of separated flow around a heavy duty vehicle (e.g. the gap between tractor and trailer, the wheel housing and the under body or the rear side) and all these areas contribute to an aerodynamic drag increase and to the creation of noise and soiling on the side windows [1]. Concerning trucks, a multitude of techniques have been developed during the years, from passive [2,3] to active [4-8] flow control strategies. Specifically, this work focuses on an active flow control (AFC) strategy to suppress the pressure induced flow separation that appears at the vertical front rounded corners of a truck cabin, generally called A-pillars. The control of turbulent separated flows is in fact one of the highly important topics in fluid mechanics [9] and the ultimate goal of this ongoing project is to implement an effective AFC able to minimize the side recirculation bubble of a truck cabin. This kind of separation resembles the separated flow around a stalled aerofoil characterized by an early stage shear layer defined by small fluid structures, and a downstream wake mainly composed by large eddies. Previous works of similar aerodynamic conditions have shown the effectiveness of a flow control device, for both static stalled [10, $11]$ and pitching $[12,13]$ aerofoils. Taking inspiration from the flow control techniques developed in this field, an AFC strategy is investigated and readapted here for ground vehicles. Studies on ground vehicle applications have approached this problem using different techniques, from suction and oscillatory blowing [14-16] to plasma actuators [17-19]. In parallel to these techniques, synthetic jet actuators have shown to be extremely effective, especially for pressure induced separating flows. This work, in particular, aims to follow up the previously published large eddy simulations (LES) [20] and experimental [7] works. In these two studies, an AFC was implemented both numerically and experimentally. Following the same path, the implementation of a more robust synthetic jet is studied further by intervening on one specific design variable: the AFC slot disposition along the A-pillar. Without any doubt, flow control based on synthetic jet actuation has a large potential but it is also very dependent on many variables involved. Principally, these variables are the magnitude and frequency of the actuation, the direction of the jet flow and the distribution and the location of the actuation slots. The focus of this paper is on the latter. The main goal is to find and alternative disposition of the actuation slot, for a more robust flow control, that it is not affected by the change of the separation location due to external conditions. Taking inspiration from the work of Vernet [19], the authors want to show an alternative slot disposition, independent from the location of the flow separation. First, the location of a standard vertical slot actuation is moved in different positions to reproduce the possibility of a flow separation happening after, before or very close to the actuation. Secondly, the effectiveness of an alternative to the vertical actuation slot (a streamwise distribute slot array) is tested. The problem is tackled by using both CFD and wind tunnel experiments. The first approach consists of LES of a simplified geometry. This study is preliminary done to verify the potential of different slot dispositions. Gathering the information from this first section, an experimental campaign is carried out on a fully three-dimensional geometry. In conclusion, the main objectives of this paper are listed below: 
- The positioning and distributions of the actuation slots has found to play a major role in the control of a pressure induced separation.

- A streamwise slot actuation is compared with three vertical slot actuations.

- An explanation of the flow mechanism describing the streamwise slot actuation is proposed.

- An experimental proof of concept, that corroborates the numerical findings, is also presented here.

The reminder of the article is organized as follows: Section 2 details the numerical and experimental set-up and the corresponding geometrical models. Section 3 is divided in two main parts: first, LES results are presented and a description of the flow control mechanism is proposed. Second, the wind tunnel data (forces measurements, particle image velocimetry (PIV) and tufts flow visualizations) are reported and discussed. Conclusions are presented in Section 4.

\section{The Numerical and Experimental Models}

Two models are used for this study. The first one (Fig. 1a, $W=2.49 \mathrm{~m}$ and $L=4 W$ ), used for LES at $R e=1 \times 10^{5}$, was previously used in [20]. In particular, a section of a simplified truck is taken into account as shown in Fig. 1a. This picture shows the simplification of the numerical domain which has been reduced to a front facing step like geometry. Boundary conditions (BC) are also described in Fig. 1a, in particular showing the use of periodic $\mathrm{BC}$ on the top and bottom surfaces ( $z$ direction) ideally reproducing an infinitely long (along $z$ ) model. In this way, the A-pillar separation flow mechanism can be isolated. The zero net mass flux jet flow was reproduced applying an inlet/outlet BC on the slot surface. This condition is defined by a sinusoidal time varying signal defined by an amplitude $U_{\text {afc }}=0.1 U_{\text {inf }}$ (with $U_{\text {inf }}=0.64 \mathrm{~m} / \mathrm{s}$ ) and a normalized frequency $F^{+}=3$. As mentioned previously, the main and only difference compared to the previous study is the slot orientation and location which have been re-adapted in the present work. Specifically regarding simulations, the streamwise slots are $0.1 \mathrm{~m}$ long, distributed on the spline generated curvature. For clarity, the spline points are also reported here (Table 1) as referred in

(a)

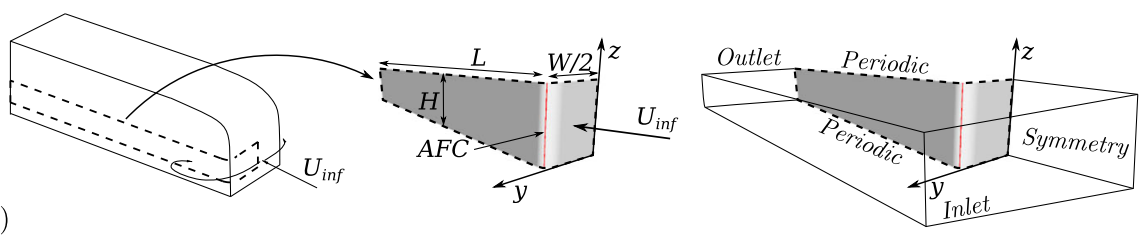

(b)

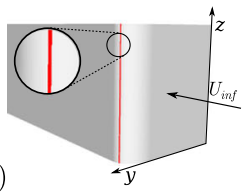

(c)

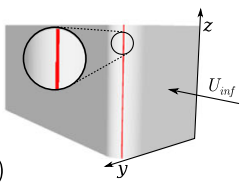

(d)

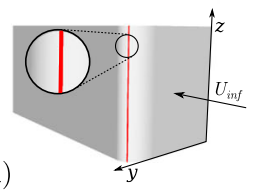

(e)

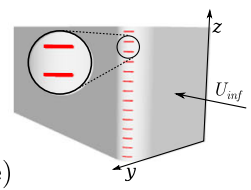

Fig. 1 The numerical domain and the slot configurations used. a The numerical test section extrapolated from a truck section. $\mathbf{b}$ The AFC slot located downstream of the flow separation point. $\mathbf{c}$ The AFC slot located upstream of the flow separation point. $\mathbf{d}$ The AFC slot located at the flow separation point. e The array of AFC slots aligned in streamwise direction 
Table $1 x$ and $y$ coordinates of points describing the curvature spline

\begin{tabular}{lll}
\hline Points & $x$ coordinate in $\mathrm{m}$ & $y$ coordinate in $\mathrm{m}$ \\
\hline$p_{1}$ & 0.000 & 0.931 \\
$p_{2}$ & 0.009 & 1.023 \\
$p_{3}$ & 0.023 & 1.070 \\
$p_{4}$ & 0.041 & 1.114 \\
$p_{5}$ & 0.065 & 1.153 \\
$p_{6}$ & 0.099 & 1.192 \\
$p_{7}$ & 0.108 & 1.200 \\
$p_{8}$ & 0.141 & 1.223 \\
$p_{9}$ & 0.190 & 1.242 \\
$p_{10}$ & 0.222 & 1.245
\end{tabular}

[20]. Their width is $0.010 \mathrm{~m}$ (the same as the vertical slots), and the number of slot is 14 . The covered area by the streamwise slots is the same as the one covered by the vertical slot since the dimension of the model in $z$ direction is $1.4 \mathrm{~m}$. The streamwise slots start at point $p_{5}=(0.065 ; 1.153) \mathrm{m}$ (Table 1$)$ and extend along the curvature for $0.1 \mathrm{~m}$.

The LES based on the standard Smagorinsky model are performed in a commercial finite volume solver AVL FIRE ${ }^{T M}$ [21]. The numerical setting, the mesh resolution and the geometrical characteristics of boundary conditions and the model used are detailed in the previously mentioned paper. Figure 1 shows the numerical model and four different dispositions of the AFC slots. Considering the vertical slot configurations, the actuation takes place downstream (Fig. 1b), upstream (Fig. 1c) and at the separation point (Fig. 1d) of the undisturbed/uncontrolled flow. The streamwise slots configuration (Fig. 1e) spans a larger section of the curvature of the A-pillar, eliminating the dependency of the separation point variable. In the latter setting, the total slot area is kept constant with respect to the vertical slot case area.

The second model (Fig. 2, with $W_{\text {exp }}=H_{\text {exp }}=0.4 \mathrm{~m}$ ), used for the experiments at $R e=5 \times 10^{5}$, was already used and described in [7]. Also in this case, a new set of A-pillars have been designed to investigate the slots location and distribution. The experimental setting, and the performance of the actuators are detailed in the mentioned experimental work. Six different slot configurations are observed experimentally. The vertical slot configuration is used in three different positions (downstream, upstream and at the separation point) as it was done for the numerical cases. Moreover, a streamwise, an alternated $45^{\circ}$ inclined and a $30^{\circ}$ inclined slots configurations are used (see Fig. 2).

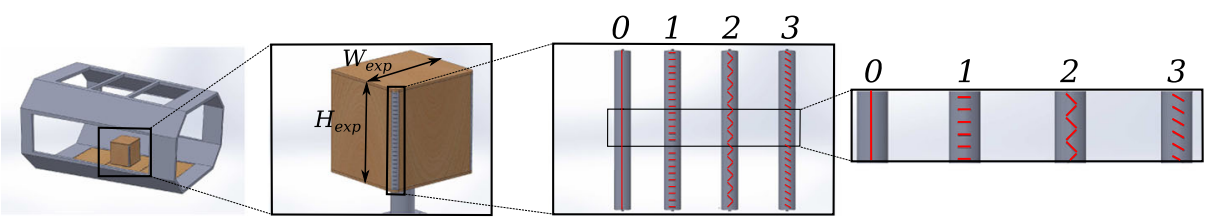

Fig. 2 The experimental model and the slot configurations used. From left, the model CAD placed in the wind tunnel test section. The model and the positioning of the A-pillar. Four different cases of actuation slots: 0 represent the spanwise slot (as for the numerical case its position is changed to downstream, upstream and on the separation point), 1 represents the array of streamwise actuation slots as used in the numerics, while 2 and 3 are two alternative variants not used in CFD 


\subsection{The momentum coefficient $C_{\eta}$}

The momentum coefficient $C_{\eta}$ is an indicator of the energy spent for the actuation $\left(\bar{I}_{j}\right)$ with respect to the energy of the unactuated flow and it is presented as follows:

$$
\begin{gathered}
\bar{I}_{j}=\left(\frac{2}{T}\right) \rho_{j} G \int_{0}^{T / 2} U_{a f c}^{2}(t) d t \\
C_{\eta}=\frac{\bar{I}_{j}}{\frac{1}{2} \rho W U_{i n f}^{2}} .
\end{gathered}
$$

Here, $G$ and $U_{a f c}$ are the width of the slots and the velocity of the slot jets. $\rho_{j}=\rho$ is the flow density and $T$ is the actuation period, while $W$ is the double width of the model, Fig. 1a. Comparing simulations and experiments, the momentum coefficient varies. In particular, the higher Reynolds number used in wind tunnel experiments $\left(R e=5 \times 10^{5}\right)$ requires a higher momentum coefficient for the actuation to be effective. Although experiments and simulations present a different value, $C_{\eta}$ is kept constant within all simulations, and unchanged for all experimental configurations. More in specific, $C_{\eta}=4 \times 10^{-5}$ is used for all simulations (both for vertical and streamwise slot cases) and $C_{\eta}^{\text {exp }}=6.25 \times 10^{-4}$ is used in the experimental campaign. All the frequencies in the present work are described in terms of the second non-dimensional parameter, the reduced frequency $F^{+}$(also called actuation Strouhal number).

$$
F^{+}=\frac{f}{U_{\text {inf }} / W}
$$

Here $f$ represents the frequency in hertz. In order for the simulations to be consistent with the experiments, $F^{+}=3$ is used for the actuation of all cases. The effect of different frequencies was previously investigated in [7] and [20] for the vertical slot configuration and it would be of great importance to study their effect in the streamwise slot case. However, investigating the influence of different frequencies would go beyond the main goal of this study, which is to investigate the main effect of orientation and position of the actuation slots. In addition, the same scaled spacing between the streamwise slots is used in both numerics (Fig. 1e) and experiments (Fig. 2 cases 1-3): $\frac{H}{N \times W}=\frac{H_{\exp }}{N_{\exp } \times W_{\exp }}$, where $N$ and $N_{\text {exp }}$ represent the number of slots of simulations and experiments, $H$ and $H_{\text {exp }}$ are the heights of the numerical and experimental models and $W$ and $W_{\text {exp }}$ are the widths of the numerical and experimental models. Concretely, a different position of the vertical slot can be related to a different yaw angle of the incoming flow. In fact when a moving vehicle experiences impinging wind at yaw, the separation point at the A-pillar changes. Likewise, the change of the vertical slot position simulates this behaviour and highlights the limits of such an actuation. Covering a larger span of the A-pillar surface, the chances to miss the separation point of the flow decreases, theoretically creating a more robust flow control.

\section{Results}

Both numerical and experimental results show a trend that highlights the benefits of the streamwise slot actuation compared to the vertical configuration. In general, a streamwise slot actuation brings two main advantages. The first one is that the mixing of the boundary layer is enhanced early upstream of the separation of the flow, the fluid mechanism behind this enhancement is described in the following subsection using LES results. The second 
is that, since the streamwise slots cover a larger portion of the model's curvature, the location of the slot does not represent a critical variable to be investigated any longer, enhancing the robustness of the present flow control. Compared to the vertical slot, where the location choice is critical for the optimization of the control, the streamwise configuration allows a larger range of separation points which will always fall within the range covered by the slots along the curvature. In this section, LES simulations are first analyzed, verifying the aerodynamic performances in terms of reduction of the recirculation bubble and drag force coefficient. Later, an interpretation of the flow mechanism created by the streamwise array of slots is attempted using vortex identification methods applied on both the average and instantaneous flow field. LES are conducted on three different vertical slot configurations in which the position of the slot is changed (Fig. 1b and c), and one streamwise slot configuration in which the orientation of the slot is modified from vertical to horizontal (Fig. 1f). In particular, the vertical slot is positioned before, after and on the separation point showing the high sensitivity of the results influenced by the slot location. The last case (streamwise slot) outperforms the other three, having, in addition, no location dependency. In the final part of this section, the findings of the LES investigation are applied to a 3D experimental model, verifying the flow control benefits in terms of aerodynamic performance.

\subsection{LES results: actuation performance and flow control mechanism at $R e=1 \times 10^{5}$}

Before detailing the LES results, it is worth to mention that a slightly different mesh construction is used for the streamwise slot configuration due to the different geometrical nature of the slots. Specifically a finer resolution is needed in the $z$ direction in order to accurately discretize the slot along their shortest dimension, which is in the $z$ direction in case of a streamwise slot actuation. This results in a 20\% larger cell count (total cell count is 6 million) and a $20 \%$ finer resolution along the $z$ direction $\left(\Delta l_{\max }^{+}=15\right)$ compared to the finest mesh used in the previous numerical study. However, the mesh construction does not affect the results. In particular, Fig. 3 shows that the two meshes predict very similar velocity pro-

(a)
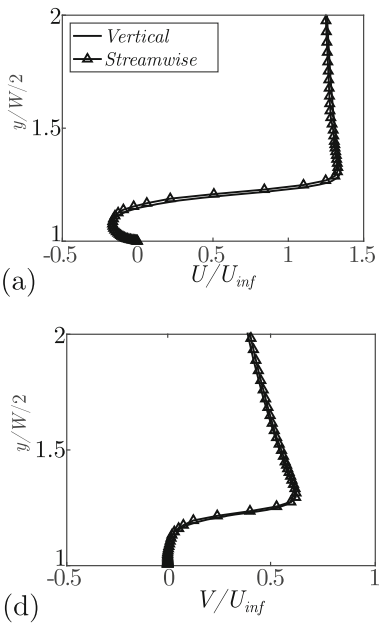
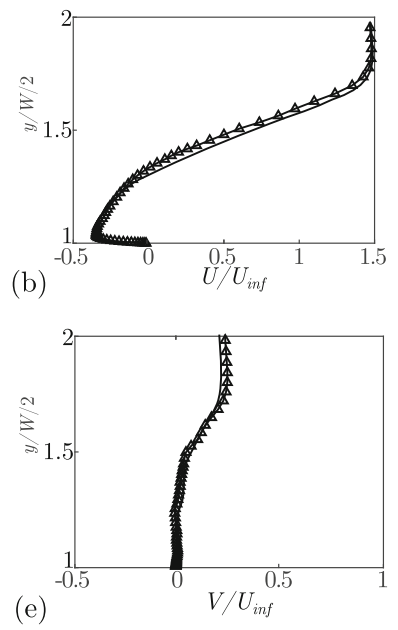
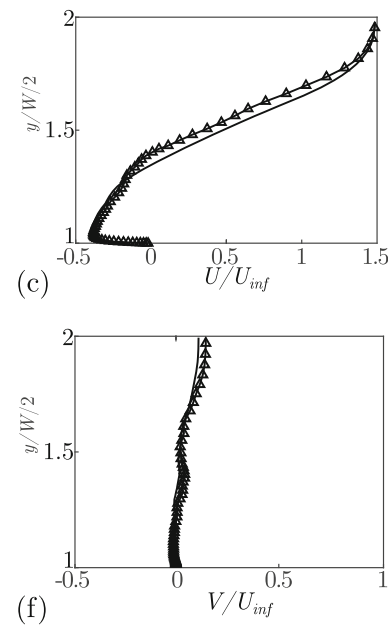

Fig. 3 A comparison between the unactuated results from the vertical slot mesh (_ $)$ and the streamwise slot mesh $(\triangle)$. (a, d) $x_{1} / \frac{W}{2}=0.48,(\mathbf{b}, \mathbf{e}) x_{2} / \frac{W}{2}=1.12,(\mathbf{c}, \mathbf{f}) x_{3} / \frac{W}{2}=1.76$. The reader is referred to Fig. 1 for the location of the origin 
files for both $U$ (a-c) and $V$ (d-f) velocity components when the flow is not actuated. A mesh resolution study was previously performed on the same geometrical model, showing grid independence for the selected resolution. The reader is referred to [20] for mesh and resolution details.

\subsubsection{The effect of location and orientation of the slot}

As said before, the control effectiveness is tested changing the location (before, after and on the separation point) and the orientation (from vertical to horizontal) of the jet slots. Figure 4 depicts the recirculation bubble and its evolution for all simulated cases, where the actuated flows are compared with the unactuated flow. Figure 4 clearly shows the advantage of having an actuated flow. The recirculation bubble size is in fact reduced in all actuated cases. For clarity, the same momentum coefficient $C_{\eta}$ is applied for all actuations, both when vertical and streamwise slots are in use (see also section 2).

Focusing particularly on the location change (only vertical slot cases Fig. 4b-d), one can notice the high sensitivity of the control to the separation point when the slots are placed vertically along the $z$ direction. A strong dependency on the actuation position is visible when the vertical slot is used (Fig. 4b-d and $\varangle, \boldsymbol{\Lambda}, \boldsymbol{\nabla}$ in (f)). Concretely, when the actuation slot is placed the closest to the natural separation point of the flow (Fig. $4 \mathrm{~d} \boldsymbol{\nabla}$ in (f)), the flow experiences the highest reduction of the recirculation bubble (74\% reduction), when related to the other vertical slot configurations. When the slot is positioned downstream (Fig. 4b, $\varangle$ in (f)) or upstream (Fig. 4c, $\boldsymbol{\Delta}$ in (f)) of the separation point, the actuation is in fact not as effective as described in the previous configuration. Specifically, it is the least effective when is placed downstream the separation. In this position the turbulent flow is

(a)

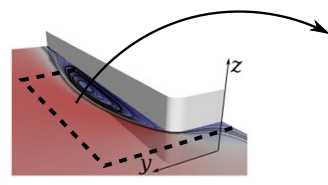

(c)

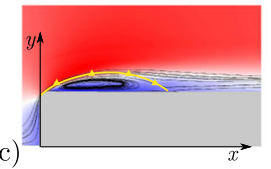

(d)

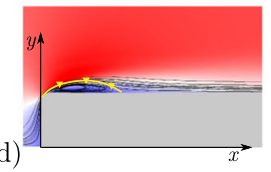

(e)

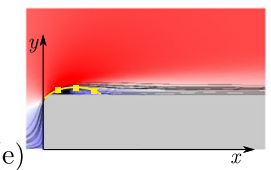

(f)

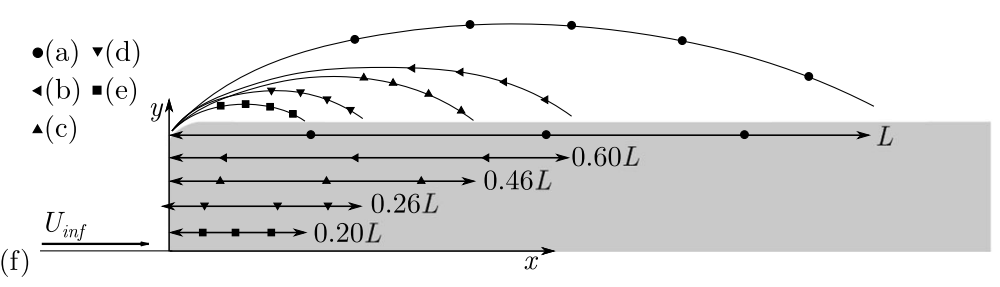

Fig. 4 The recirculation bubble reduction. a Unactuated flow. b Actuated flow with a vertical slot located downstream of the flow separation point. c Actuated flow with a vertical slot located upstream of the flow separation point. d Actuated flow with a vertical slot located on the flow separation point. e Actuated flow with streamwise slots. f A sketch that quantifies the recirculation bubble reduction. $\bullet$ unactuated, $\boldsymbol{\Delta}$ vertical slot upstream separation, $\varangle$ vertical slot downstream separation, $\boldsymbol{\nabla}$ vertical slot on separation, $\mathbf{\square}$ streamwise slots. The lines in (a-e) are coloured in yellow for clarity, later they are all overlaid in (f) and coloured in black 
already separated and a larger momentum coefficient would be necessary for the control to keep the flow attached to the surface and perform an effective pressure recovery to stabilize the separated region again. Nevertheless, it is worth to mention that even in the worst case scenario (Fig. $4 \mathrm{~b}, 4$ in (f)) a $40 \%$ reduction of the length of the recirculation bubble is observed.

Moving our focus on the streamwise slot actuation, this brings an improvement of $80 \%$ in terms of bubble length reduction. Therefore, the streamwise slots solution (Fig. 4e, $\mathbf{\square}$ in (f)) achieves the most performant result. In addition, the size of the recirculation bubble, presented in Fig. 4f, well relates to the $C_{d}$ values presented in Table 2. For clarity, the numerical $C_{d}$ and experimental $C_{d}^{\exp }$ coefficients of drag are defined as follows:

$$
C_{d}=\frac{F_{x}}{0.5 \rho U_{i n f}^{2}(W / 2) H} ; \quad C_{d}^{\exp }=\frac{F_{x}}{0.5 \rho U_{i n f}^{2}\left(W_{\text {exp }}\right) H_{\text {exp }}} .
$$

By evaluating this parameter, the streamwise slot configuration outperforms all other configurations in absolute value but also in robustness of the method, since a small variation of the vertical slot positioning brings a large change of the total $C_{d}$ value.

\subsubsection{Best vertical slot vs. streamwise slot actuation}

It is interesting to investigate deeper the flow mechanism that describes the streamwise slot configuration. The flow mechanism describing the vertical slot model was already explained in [20] and it was found that the actuation is effective only when the actuation frequency matches the natural frequencies of the flow. In the vertical slot configuration, the actuation interacts with the natural frequencies of the flow energizing or suppressing certain flow structures. Specifically, it was found that the optimal actuation frequency was the one corresponding to the natural shear layer separation frequency. When the slots are placed in the streamwise direction, the nature of the control intrinsically changes. More in detail, the latter configuration forms couples of counter rotating vortices that develop in the streamwise direction creating a swirl in the flow which in turn energizes and enhances the mixing of the boundary layer. For clarity, the effect of the actuation frequency, in this particular case, has not been verified and the authors retain this investigation as a natural continuation of the project. The actuation frequency was chosen to create a comparable case with the best case obtained in the vertical slot configuration. A series of pictures of the phase averaged flow are presented to describe this behaviour. Figure 5 shows the evolution of positive (red) and negative (blue) isosurfaces of streamwise vorticity. Alternated isosurfaces of negative and positive vorticity indicate the presence of counter swirling vortices forming along the streamwise slots. It is interesting to note that these areas do not only change sign from one side to the other of the slot but a periodic change over time is also observed. In other words, layers of opposite sign vorticity forms on both sides of the slot, periodically. A jet flow normally produces a pair of counter rotating vortices that in presence of cross flow develop and enlarge along the streamwise direction $[22,23]$. Here, the periodic blowing and suction

Table 2 Averaged $C_{d}$ values of different numerical configurations. $V_{d o w n}$ and $V_{u p}$ and $V_{o n}$ stand for vertical AFC positioned downstream, upstream and on the separation point, respectively. $S$ stands for streamwise AFC

\begin{tabular}{lllll}
\hline AFC off & $V_{\text {down }}$ & $V_{u p}$ & $V_{\text {on }}$ & $\mathrm{S}$ \\
\hline 0.67 & 0.58 & 0.49 & 0.41 & 0.33 \\
\hline
\end{tabular}



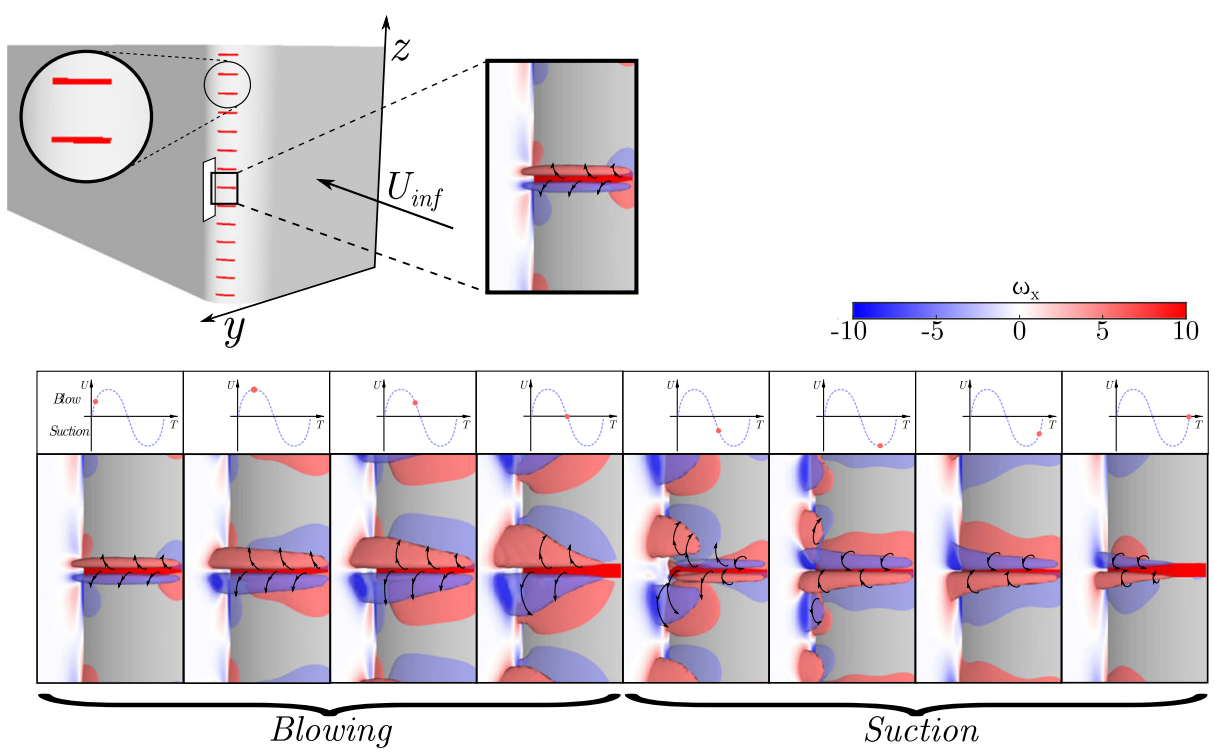

Fig. 5 The temporal evolution of the vortical structures generated by the actuation. Two vorticity isosurfaces at $\omega=-5$ (blue) and $\omega=5$ (red) are plotted. The top line shows a zoom of one slot for a better understanding of the bottom line. The latter shows the phase averaged results of the structures at eight instant along the actuation signal

naturally arises the formation of two main pairs of structures with opposite signs. The first pair grows, during the blowing period, enlarging from the beginning to the end of the slot. The second, generated by the suction effect, stays confined, near and elongated along the slot edges. These results recall the experimental study performed by Jukes et al. [24], where the formation of positive and negative vorticity layers was observed. In the cited work, the authors distinguish the lower and upper shear layer visualized by flow averaged results. The present phase averaged analysis is therefore necessary to shed light on the vorticity stratification effect mentioned in [24]. Specifically, the phase averaged analysis (Fig. 5) highlights the absence of vorticity stratification. This figure rather clarifies the continuous evolution of two counter rotating pair of structures, that might mislead to the interpretation of a stratified phenomenon if only the ensemble average of all phases is taken into account. Considering the vertical slot separation control mechanism it is worth mentioning that, in comparison to the $3 \mathrm{D}$ mechanism described above, it reduces the control to a 2D like separation control mechanism where only vorticity in $z$ direction is generated periodically with the oscillation of the actuation signal.

Evaluating the larger effect of the actuation and taking a broader look at the structures formed in the flow by different slots, it is possible to appreciate the formation of a much more fluctuating flow when the streamwise slots are used. This is measured in terms of instantaneous structures (Fig. 6) and averaged Reynolds stress (Fig. 7). Specifically, the isosurfaces of the second invariant of the velocity $Q$ [25] are shown in Fig. 6. This picture shows the formation of much larger structures when a vertical slot is used. As mentioned earlier, the use of a vertical slot allows the formation of a $2 \mathrm{D}$ like separation having a more homogeneous separation control all along the $z$ direction. Using streamwise jets, the separation control mechanism takes a 3D connotation as it was also visualized in Fig. 5. 
(a)
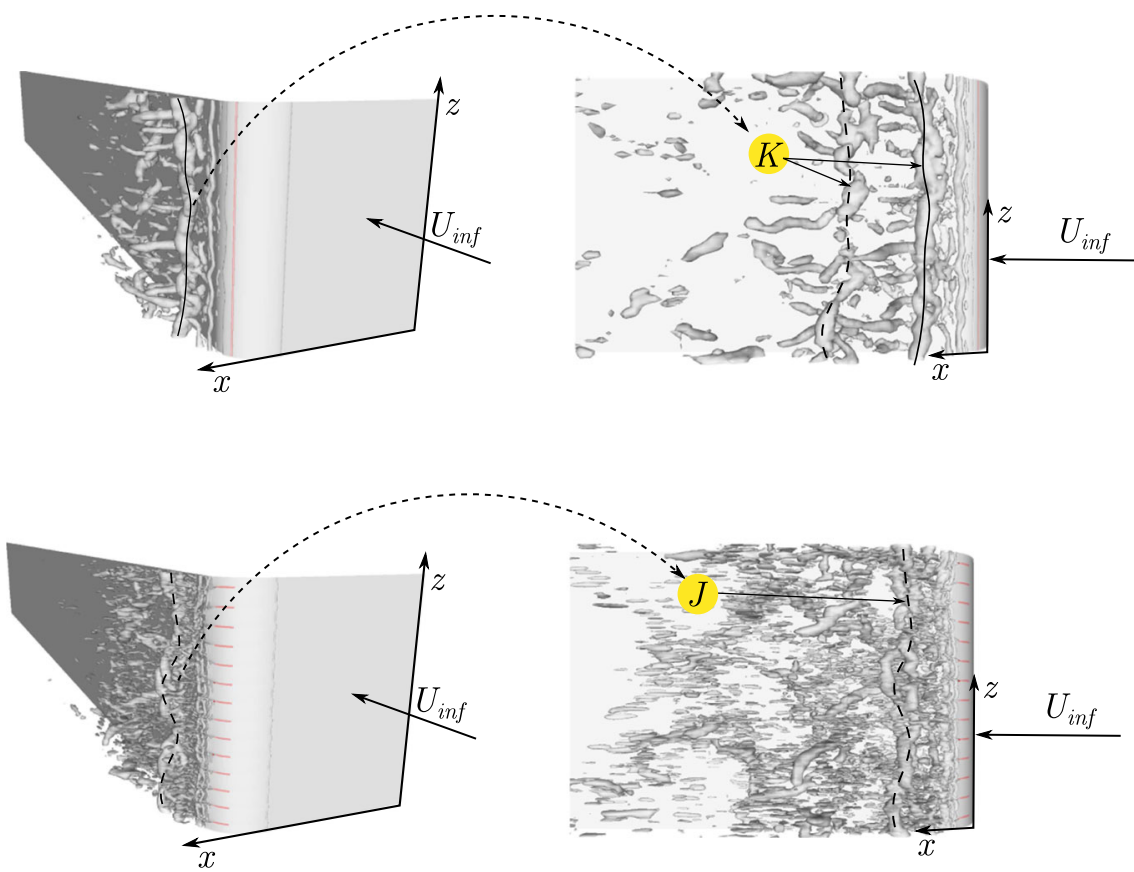

Fig.6 $Q$ isosurface for the vertical and the streamwise slot actuation $\left(Q=80 s^{-2}\right)$. a shows the vertical slot configuration (slot on the separation point) while b shows the streamwise slots configuration

Figure 6a presents the formation of one of the mentioned large structures (structure $K$ ) which tends to span the entire domain height ( $z$ direction), recursively. Figure 6a shows the early formation of $K$, after the slot interaction with the incoming flow (( -$)$ in Fig. 6a), and its rapid dissipation into the mainstream ((- $)$ in Fig. 6a). Much more complicated is to reconstruct the presence of a well organized vortical structure when streamwise actuators are in use. In this case, the large structure , (_ - ) in Fig. 6b, appears disconnected into smaller vortices, and a multitude of finer sctructures describes the flow downstream the separation. These smaller eddies (Fig. 6b), as a consequence, increase the level of turbulence in the flow. This last aspect is supported by the time averaged isosurfaces of the streamwise Reynolds
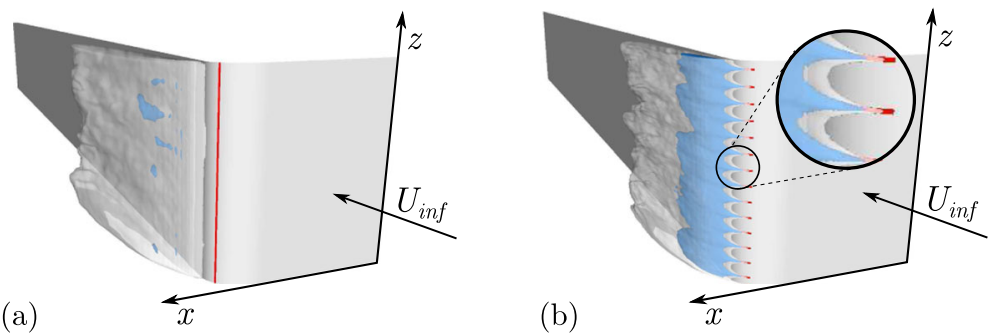

Fig. 7 Streamwise Reynolds normal stress $\left(\left\langle u_{x}^{\prime 2}\right\rangle\right)$ isosurface for the vertical and the streamwise slot actuation. Blue: $\left\langle u_{x}^{\prime 2}\right\rangle=0.12$. White: $\left\langle u_{x}^{\prime 2}\right\rangle=0.05$. a shows the vertical slot configuration (slot on the separation point) while b shows the streamwise slots configuration. b contains a zoom to highlight the tubercles formation at the rounded edge 
normal stress $\left(\left\langle u_{x}^{\prime 2}\right\rangle\right)$ depicted in Fig. 7. Figure 7a shows in fact the presence of a smaller region of high intensity stress (light blue isosurface) compared to Fig. 7b. Moreover, the normal stress isosurfaces in Fig. 7b show a tubercled formation at the leading edge of the model (see the zoom in the circle). The streamwise actuation seems to recreates a virtual tubercled leading edge surface which its beneficial effect on stalled aerofoils are studied, among others, in [26-28].

The energy of the flow necessary to win the adverse pressure gradient, created by the front curvature, can be measured by looking at the streamwise velocity profiles collected along the rounded edge of the model. The higher streamwise velocity close to wall, the easier is for the flow to win the adverse pressure gradient, delay separation and reduce the recirculation bubble. Bearing this in mind, the two most effective actuations are compared in Fig. 8. This picture shows a comparison of the tangential velocity at different locations. The enhanced boundary layer mixing created by the streamwise slot is visible since the early stage of the actuation (Fig. 8b) and becomes even more visible along the development of the flow. In fact, the streamwise actuation forms fuller velocity profiles at all locations along the rounded edge, Fig. 8b-f. Nevertheless, both the actuation typologies develop fuller velocity profiles along the curvature of the model when compared to the unactuated case.

\subsection{Experimental results: proof of concept at $R e=5 \times 10^{5}$}

The results collected in the previous section are further used as an input to recreate a proof of concept case that shows the effectiveness of streamwise slots at a higher Reynolds number. The model chosen for the experiments was the one used in previous experimental [7] and numerical $[8,29]$ works. Tufts visualizations and PIV, supported by force measurements, are used to evaluate the effectiveness of different control solutions. Tufts were used to visualize the flow behaviour on the surface of the model and PIV was used to observe the

(a)
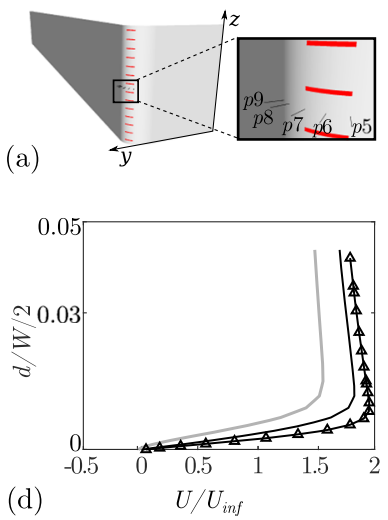
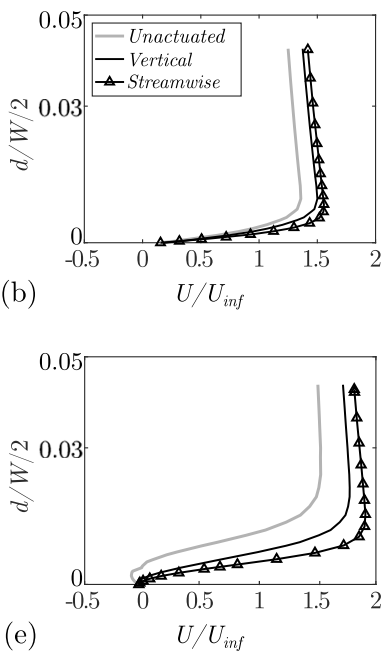
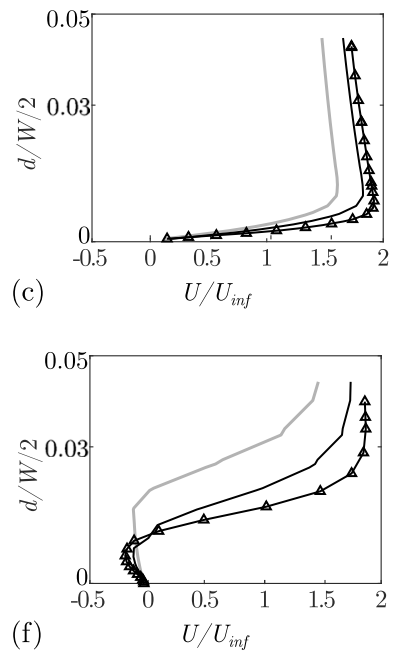

Fig. 8 Tangential velocity to the surface of the model at different locations. Comparison between unactuated results ( $\_$), the best vertical slot actuation (_) and the streamwise slot actuation $\left(\triangle_{-}\right)$. a The profile locations. b $p 5$, c $p 6$, d $p 7$, e $p 8$ and $\mathbf{f} p 9$. The $p$ points that identify the surface location of the profiles refer Table 1, and indicate the origin of each normal profile on the model's surface. $d$ is the distance from the surface in the normal direction 
averaged recirculation bubble size. Generally, the experimental data confirm the trend found in CFD. Figure 9a shows the model placed in the wind tunnel, the red rectangle indicates the observed surface covered by nylon tufts. Figure $9 \mathrm{~b}$ shows a single frame visualization and the average tuft movement is shown in Fig. 9c. 500 single frames were averaged to obtain the result presented in Fig. 9c. Later, every averaged picture was post-processed to obtain the direction of the flow indicated by the average tuft movement distribution. The script, specifically, analyzes the average picture in the following way: (i) two surrounding circles (inner and outer, see dashed yellow lines in Fig. 9c) are drawn around the centre $C$ of each tuft sticker. (ii) The intensity of each pixel lying on the circles are evaluated and the location of the highest intensity pixel for each circle is stored. (iii) The two high intensity pixels are connected and the angle formed with respect to the $x$ direction is identified. (iv) A 2D field composing the direction of each tuft tail is stored and plot by means of averaged surface streamlines. The script cannot give quantitative values of the intensity of the velocity near the surface but only information regarding the direction of the flow is recorded. For this reason, Fig. 10 should be only considered as a qualitative representation of the model surface streamlines. The first observation from Fig. 10 is that the actuation of the flow is always beneficial in terms of recirculation bubble reduction. More in detail, a very similar result is achieved when either the vertical slot is placed on the separation point, or the streamwise slots are employed. By positioning the vertical slot actuation downstream (Fig. 10b) or upstream (Fig. 10c) of the separation point, a less effective actuation is observed and the reverse flow region on the model's surface is larger, in line with the previous LES results.

Even though the results achieved by the best vertical slot and the streamwise slot configurations are very similar (Fig. 10d and e), Figs. 11 and 12 want to stress the advantage of a streamwise slot control device compared to a position dependent vertical slot. As said in

(a)
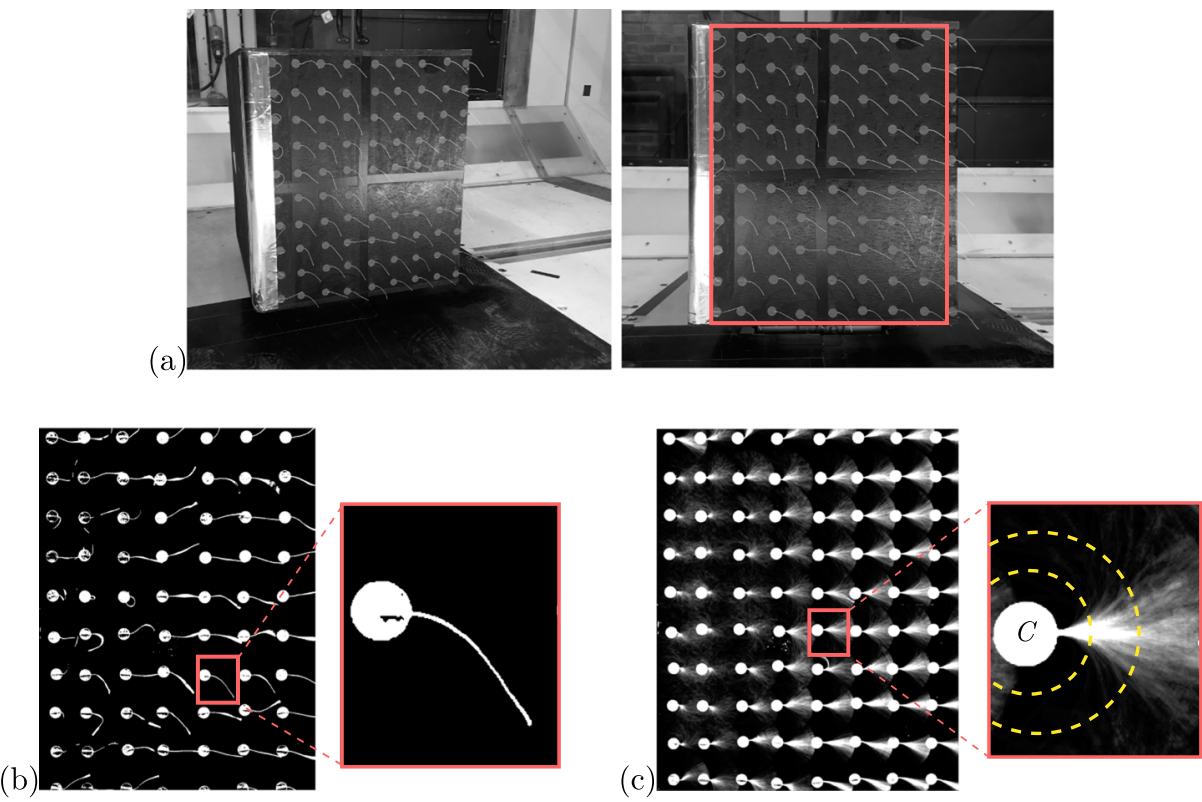

Fig. 9 Surface tuft visualizations on the side face of the experimental model. a The model placed in the wind tunnel, the red rectangle indicates the observed area. b One single frame during data collection. $\mathbf{c}$ Picture of the average movement of every tuft 
(a)

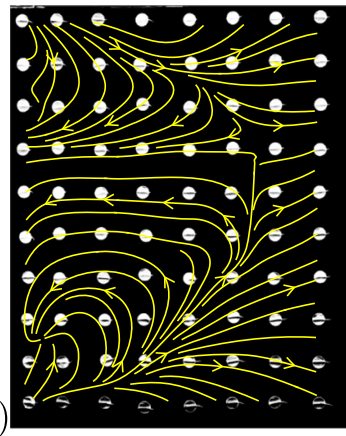

(b)
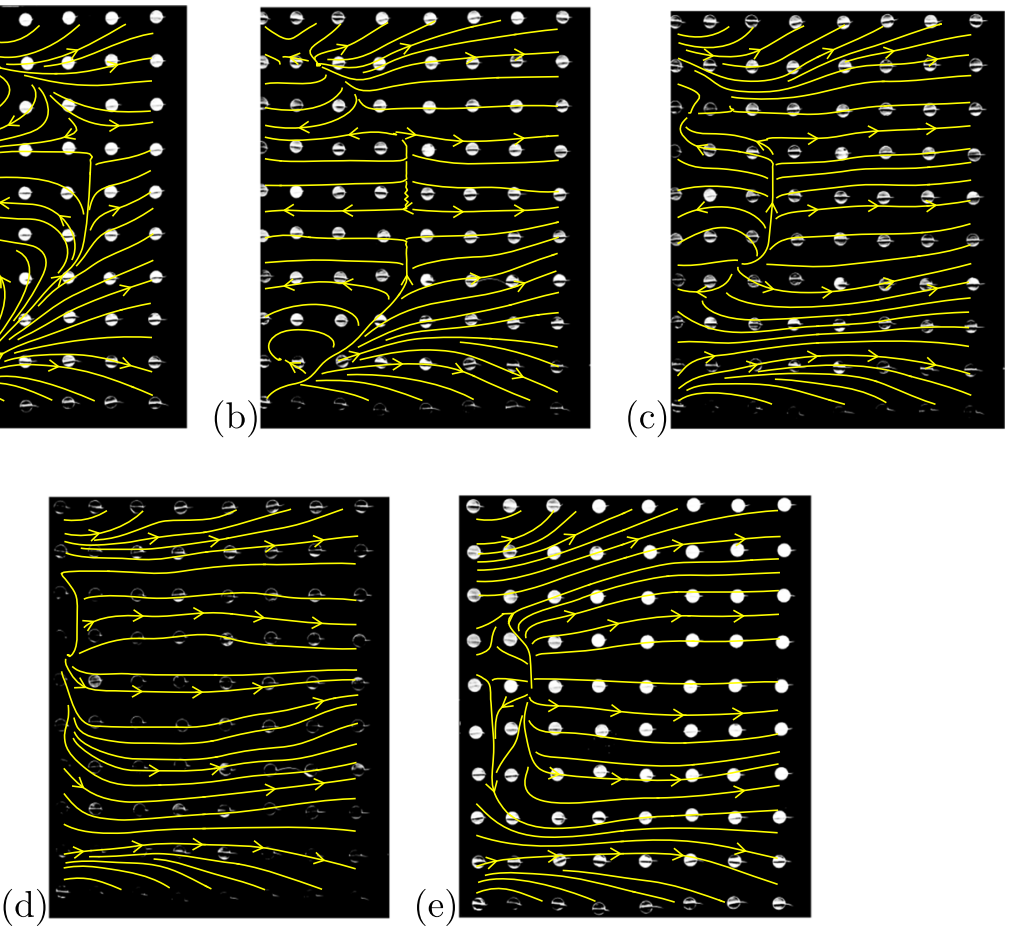

Fig. 10 Surface streamline visualizations. a Unactuated flow. b AFC slot located downstream of the flow separation point. c AFC slot located upstream of the flow separation point. d AFC slot located at the flow separation point. e AFC slots aligned in streamwise direction. Flow from left to right

Section 2, it is of major importance to overcome all possible external flow conditions. As a consequence, a vertical slot solution, which strongly relies on the optimal slot positioning, is definitely inferior compared to a configuration which is independent of the separation point of the flow, which is originally defined by the incoming yaw angle. Figure 11a shows a sketch of the model and the PIV observed area, while Fig. 11b-f show the recorded and averaged PIV snapshots for the unactuated (b), vertical slot actuated (c-e) and streamwise slot actuated (d) flows. Specifically, Fig. 11c shows the slot-after-separation case (worst vertical slot configuration) and (d) shows the slot-on-separation case (best vertical slot configuration). Figure 11e shows the global average flow of the three vertical slot positioning (slot before, after and on separation). From this picture, it is clear that the streamwise solution outperforms the vertical slot solution, giving a reliable, robust and overall better performing actuation. Three streamwise velocity profiles are plotted in Fig. 12 to corroborate the latter statement, showing consistent and fuller velocity profiles in favour of the streamwise configuration $(\triangle)$, when compared to the three-position-average (Fig. 11e and $(-)$ in Fig. 12) result. Remain anyway worth mentioning that the best vertical slot configuration ( - ) in Fig. 12 gives very similar velocity profiles to the streamwise slot solution and that the slot-after-separation configuration (_) is far worse compared to all other cases.

Aerodynamic drag values (see Table 3 ) are strongly correlated to the size of the side recirculation bubble. For all controlled cases, a drag decrease, compared to the baseline, is reported and more than $10 \%$ drag reduction is achieved when the streamwise slots are 
(a)

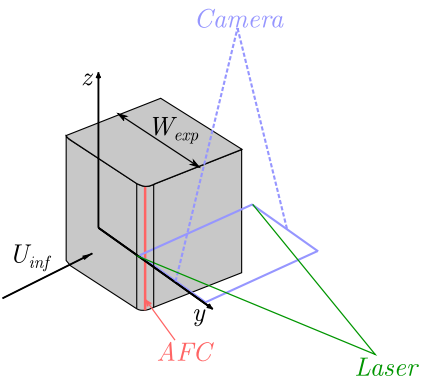

(c)

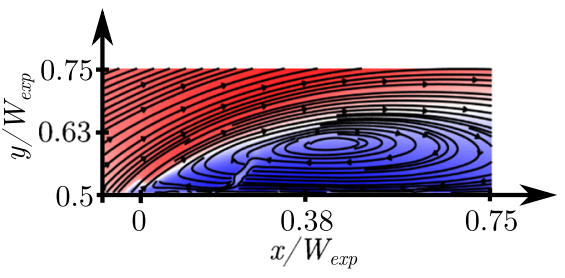

(b)

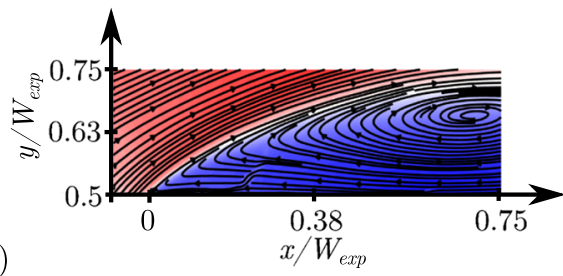

(d)

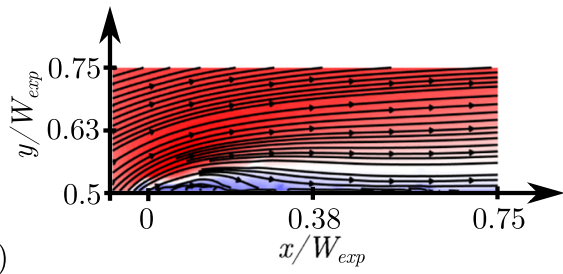

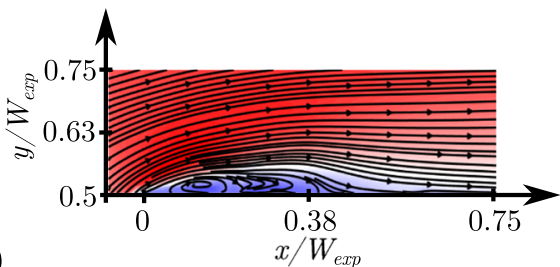

(e)

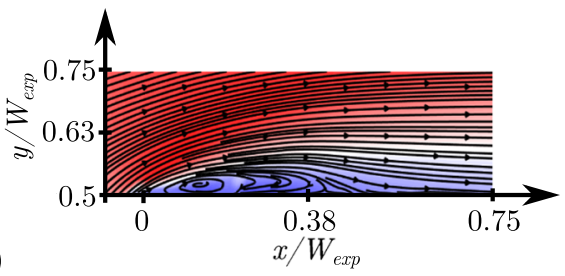

(f)

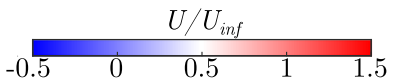

Fig. 11 PIV observation of the flow. a A sketch of the PIV observed region. b The unactuated recirculation bubble. $\mathbf{c}$ The recirculation bubble of the vertical slot positions, slots after the separation point. $\mathbf{d}$ The recirculation bubble of the vertical slot positions, slots on the separation point. $\mathbf{e}$ The recirculation bubble averaged over the actuation of the three different vertical slot positions. $\mathbf{f}$ The recirculation bubble of the streamwise slot actuation

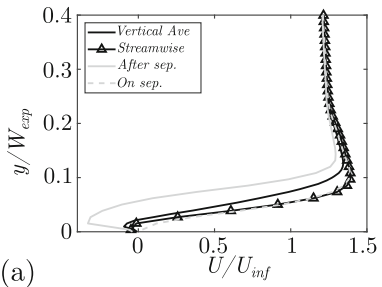

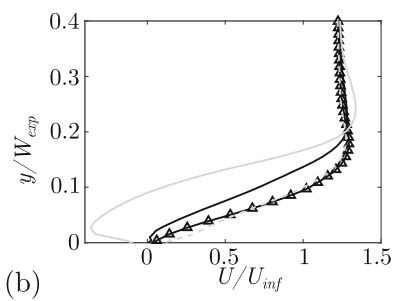

(c)

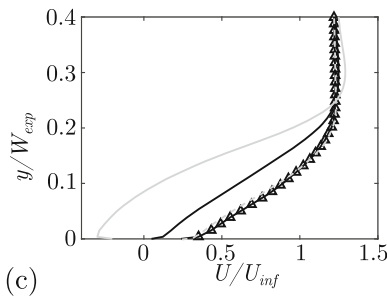

Fig. 12 The streamwise velocity profile comparison between the three-vertical-averaged actuation (—), the vertical slot after separation ( - ), the vertical slot on the separation point $\left(-_{-}\right)$and the streamwise actuation $(\triangle)$. a $x / W=0.25$. b $x / W=0.45$. $\mathbf{c} x / W=0.75$ 
Table 3 Averaged $C_{d}$ values of different experimental configurations. The numbers in brackets correspond to Fig. 2 variations. A, B and C refer to the positioning of the vertical slot model. In particular they correspond to downstream, upstream and on the separation point, respectively

\begin{tabular}{lllllll}
\hline AFC off & $(0) \mathrm{A}$ & $(0) \mathrm{B}$ & $(0) \mathrm{C}$ & $(1)$ & (2) & $(3)$ \\
\hline 1.01 & 0.95 & 0.91 & 0.90 & 0.90 & 0.93 & 0.93 \\
\hline
\end{tabular}

employed. In addition, the inclined streamwise slot variants (see Fig. 2 variants 2 and 3) results in a lower drag reduction compared to the streamwise slot configuration.

At this point, a qualitative comparison of the experimental and CFD results can be made. Two things can be pointed out from this. The first one is that the vertical slot-after/beforeseparation configurations are consistently worse than the on-separation case. Comparing Fig. 4b against Fig. 11c one can observe the worst (controlled) case scenario, when the vertical slot is placed after the separation point for both CFD and experiments. The second thing is that both the on-separation vertical slot case and the streamwise case give very similar results, showing that the streamwise actuation is not worse than the optimal vertical actuation. A deeper and quantitative comparison between numerical and experimental result would be difficult at this stage. The different geometry, the different momentum coefficient $C_{\eta}$, the strong three dimensionality introduced by the experimental model, the different curvature of the A-pillar and the different $R e$ make a full comparison impossible. Nevertheless, such a comparison goes beyond the scope of this paper which aims to verify different control strategies tested at low $R e$ in CFD, using an experimental proof of concept. For completeness, the measured aerodynamic forces are also reported in Table 3 showing a $10 \%$ drag reduction in favor of the optimal vertical control and the streamwise slot configuration. The maximum force measurement uncertainty recorded during the experiment lies within $\pm 0.6 \%$ in terms of $C_{d}$ values.

\section{Conclusions}

LES and wind tunnel experiments were conducted to investigate the effectiveness of different actuation slots to control the separation of the flow at the A-pillar of a simplified truck model. As mentioned in the introduction chapter, the work is a follow up of two previous works [20] and [7] and it aims to integrate these previous studies in the research of an optimal actuation system. Both numerics and experiments show the same trend, highlighting the benefit of having a streamwise slot actuation. Using this slot typology, the flow control mechanism drastically changes and, most importantly, the location of the slot related to the separation point is no longer a critical variable in the control strategy. The average and instantaneous LES results are analysed showing the formation of a more complex flow mechanism when streamwise actuation is employed. A first explanation of the flow control mechanism is attempted showing similarities and novelties compared to a previous study on the same topic [24]. What was observed with LES results was that the location and the distribution of the actuation slots plays a crucial role toward the control of separated flows. LES also showed the overall flow behaviour, and the beneficial effect, in terms of recirculation bubble reduction, was quantified. Specifically, the streamwise slot actuation achieves $80 \%$ reduction of the length of the recirculation bubble. Moving to the experimental side, thanks to the proof of concept model, a first upscaling of the actuation was achieved increasing five times the Reynolds number and applying the control to a fully 3D bluff body. Overall, 
the use of the streamwise actuation shows the possibility to disregard the crucial positioning of a vertical slot, resulting in a more robust and globally more effective actuation strategy. Future studies will focus on the development of the actuation signal. Concretely, a multifrequency, multiactuator control is currently under development. The final goal is to develop a fully automated flow control aided by machine learning techniques.

Acknowledgements This work is funded by the Swedish Energy Agency and supported by Volvo Trucks. Software licenses were provided by AVL List GMBH. The computations were performed on resources provided by the Swedish National Infrastructure for Computing (SNIC) at LiU. M. Tokarev thanks for partial financial support given within the state contract with IT SB RAS.

Funding Information Open access funding provided by Chalmers University of Technology.

Compliance with Ethical Standards This study was funded by the Swedish Energy Agency (Energimyndigheten) with grant number 2013-003608.

Conflict of interests The authors declare that they have no conflict of interest.

Open Access This article is distributed under the terms of the Creative Commons Attribution 4.0 International License (http://creativecommons.org/licenses/by/4.0/), which permits unrestricted use, distribution, and reproduction in any medium, provided you give appropriate credit to the original author(s) and the source, provide a link to the Creative Commons license, and indicate if changes were made.

\section{References}

1. Schuetz, T.C.: Aerodynamics of Road Vehicles, Fifth Edition. SAE International (2015)

2. Cooper, K.R.: The effect of front-edge rounding and rear-edge shaping on the aerodynamic drag of bluff vehicles in ground proximity. In: SAE Technical Paper Series, vol. 1 (2010)

3. Cooper, K.R.: Truck Aerodynamics Reborn - Lessons from the Past. SAE Technical Paper Series, vol. 1 (2010)

4. Seifert, A., Shtendel, T., Dolgopyat, D.: From lab to full scale Active Flow Control drag reduction: How to bridge the gap? J. Wind Eng. Ind. Aerodyn. 147, 262-272 (2015)

5. McNally, J., Fernandez, E., Robertson, G., Kumar, R., Taira, K., Alvi, F., Yamaguchi, Y., Murayama, K.: Drag reduction on a flat-back ground vehicle with active flow control. J. Wind Eng. Ind. Aerodyn. 145, 292-303 (2015)

6. Vernet, J.A.: Plasma actuators for separation control on bluff bodies. PhD Thesis, Mechanics, School of Engineering Sciences (SCI) KTH (2017)

7. Minelli, G., Hartono, E.A., Chernoray, V., Hjelm, L., Krajnović, S.: Aerodynamic flow control for a generic truck cabin using synthetic jets. J. Wind Eng. Ind. Aerodyn. 168, 81-90 (2017)

8. Minelli, G., Krajnović, S., Basara, B.: A flow control study of a simplified, oscillating truck cabin using PANS. J. Fluids Eng. 140(12), 121101 (2018)

9. Cho, M., Choi, S., Choi, H.: Control of flow separation in a turbulent boundary layer using time-periodic forcing. ASME J. Fluids Eng. 138(c), 1-10 (2016)

10. Gad-el Hak, M., Pollard, A., Bonnet, J.P.: Flow control: Fundamentals and Practices. No. v. 53 in Flow control: Fundamentals and Practices. Springer, Berlin (1998)

11. Glezer, A., Amitay, M., Honohan, A.M.: Aspects of low- and High-Frequency actuation for aerodynamic flow control. AIAA J. 43, 1501-1511 (2005)

12. Woo, G.T.K., Glezer, A.: Transitory control of dynamic stall on a pitching airfoil. Notes on Numerical Fluid Mechanics and Multidisciplinary Design 108, 3-18 (2010)

13. Yen, J., Ahmed, N.A.: Parametric study of dynamic stall flow field with synthetic jet actuation. ASME J. Fluids Eng. 134(7), 071106 (2012)

14. Gad, L., Avraham, S.: Flow control applied to the front rounded edge of a bluff body. International Journal of Flow Control 6, 21-42 (2014) 
15. Lubinsky, G., Seifert, A.: Suction and oscillatory blowing applied to the rounded front edges of a square prism BT. In: Theofilis, V., Soria, J. (eds.) Instability and Control of Massively Separated Flows. Proceedings of the International Conference on Instability and Control of Massively Separated Flows, held in Prato, Italy, from 4-6 September 2013, pp. 157-162. Springer International Publishing, Cham (2014)

16. Seifert, A., Dayan, I., Horrell, C., Grossmann, J., Smith, A.: Heavy Trucks Fuel Savings Using the SaOB Actuator BT - The Aerodynamics of Heavy Vehicles III: Trucks, Buses and Trains, pp. 377-390. Springer International Publishing, Cham (2016)

17. Vernet, J.A., Örlü, R., Alfredsson, P.H.: Separation control by means of plasma actuation on a half cylinder approached by a turbulent boundary layer. J. Wind Eng. Ind. Aerodyn. 145, 318-326 (2015)

18. Michelis, T., Kotsonis, M.: Flow control on a transport truck side mirror using plasma actuators. ASME J. Fluids Eng. 137(11), 111103 (2015)

19. Vernet, J.A., Örlü, R., Alfredsson, P.H.: Flow separation control behind a cylindrical bump using dielectric-barrier-discharge vortex generator plasma actuators. J. Fluid Mech., pp. 852-879 (2017)

20. Minelli, G., Krajnović, S., Basara, B., Noack, B.R.: Numerical investigation of active flow control around a generic truck A-Pillar. Flow Turbul. Combust. 97(4), 1235-1254 (2016)

21. AVL, Fire manual v2014 (2014)

22. Cortelezzi, L., Karagozian, A.R.: On the formation of the counter-rotating vortex pair in transverse jets. J. Fluid Mech. 446, 347-373 (2001)

23. Karagozian, A.R.: The jet in crossflow. Phys. Fluids 10, 26 (2014)

24. Jukes, T.N., Choi, K.S.: On the formation of streamwise vortices by plasma vortex generators. J. Fluid Mech. 733, 370-393 (2013)

25. Hunt, J.C.R., Wray, A.A., Moin, P.: Eddies, streams, and convergence zones in turbulent flows. Studying Turbulence Using Numerical Simulation Databases 2: Proceedings of the Summer Program 1988 (1989)

26. Hansen, K.L., Rostamzadeh, N., Kelso, R.M., Dally, B.B.: Evolution of the streamwise vortices generated between leading edge tubercles. J. Fluid Mech. 788, 730-766 (2016)

27. Wei, Z., New, T.H., Cui, Y.D.: Aerodynamic performance and surface flow structures of leading-edge tubercledtapered swept-back wings. AIAA J. 56(1), 1-9 (2017)

28. Pérez-Torró, R., Kim, J.W.: A large-eddy simulation on a deep-stalled aerofoil with a wavy leading edge. J. Fluid Mech. 813, 23-52 (2017)

29. Minelli, G., Adi Hartono, E., Chernoray, V., Hjelm, L., Krajnović, S., Basara, B.: Validation of PANS and active flow control for a generic truck cabin. J. Wind Eng. Ind. Aerodyn. 171, 148-160 (2017)

Publisher's Note Springer Nature remains neutral with regard to jurisdictional claims in published maps and institutional affiliations. 